\title{
PREVALENCE OF COGNITIVE DISTORTIONS AMONG ADOLESCENTS IN PUNJAB, PAKISTAN
}

\author{
Sobia Ishrat ${ }^{*}$ \& Sajida $\mathrm{Naz}^{\dagger}$
}

\begin{abstract}
This study investigated the prevalence of cognitive distortions among adolescents in Pakistan. 1258 participants recruited through purposive sampling, falling in the age range of 16-22 years and studying in different Public sector collegesof Rawalpindi Division were administered the How I Think Questionnaire. The participants were placed into the following three categories; non-clinical, borderline and clinical range based on their responses on the 'HIT-Q. This Questionnaire measures four types of cognitive distortions namely minimizing / mislabeling, self-centered, assuming the worst and blaming others. Results of the study revealed that of $17.17 \%(216 / 1258)$ of the participants were identified in in the clinical category on self-centered subscale whereas $13.83 \%$ (174/1258) were in the clinical category of Blaming others subscale..48 \% (148/1258) responses were in the clinical category on the "assuming the worst" subscale and $14.1 \%$ in the minimizing/ mislabeling subscale. 3.00-4.89 was the clinical range for the subscale of minimizing/mislabeling and Clinical range for "assuming the worst" subscale according to manual of the HIT-Q was 3.004.92. Meanwhile responses also indicated that $31.8 \%$ of adolescents scored high on oppositional defiance. $37.1 \%$ in physical aggression and $60 \%$ in stealing. Results suggest that adolescents in Pakistan has severe problem of thinking in distorted way and there is dire need for counselling centers to be established in every college in Punjab Pakistan.
\end{abstract}

Keywords: adolescents, assuming the worst, blaming others, minimizing/ mislabeling, cognitive distortions, self-centered, Pakistan.

\footnotetext{
* PhD Psychology, Department of Behavioural Sciences, Fatima Jinnah Women University, Rawalpindi, Pakistan.

$\dagger$ Assistant Professor, Department of Behavioural Sciences, Fatima Jinnah Women University, Rawalpindi, Pakistan.
} 


\section{Introduction}

Decision making plays an important role in life. Decisions are made based on thinking patterns. It is the nature of human being to give meanings to actions of ourselves and others. Humans evaluate and interpret the contextual meaning of their experiences. These interpretations of incoming experiences and attributing these actions have an intricate relationship with each other for example the judgement about the surrounding world helps human being in setting beliefs in the minds that are kept in mind for a long time along with the values, which consequently result in evaluation of those beliefs, values and conduct from the individuals and groups in society (Helmond, Overbeek, Gibbs \& Brugman, 2015). Thinking errors make an individual to either selfnegate or self-degrade themselves. These cognitive distortions or errors in thinking are caused by faulty and ineffective information processing resulting from inconsistent, irrational anderroneous way of thinking (Rehna, Hanif \& Aqil, 2020).Cognitive distortions or errors in the thinking process can then be referred as those actual mechanisms or procedures that result in biased judgement or evaluations (Beck \& Weishaar, 1989).

These distortions in the thinking patterns can be "criminogenic" as these can be used by an individual to protect the self from blame by detaching themselves from their maladaptive behaviors by blaming others (Barriga, Landau, Stinson, Liau \& Gibbs, 2000). They can also be used to avoid negative self-concept. These distorted thinking patterns are consequently used to reinforce antisocial behavioral patterns (Barriga, Landau, Stinson, Liau \& Gibbs, 2000).

The tendency in human nature to attribute all the negative aspects (negative schemas) and events to the self and all the positive aspects and events to outside world (Stewart, Kennard, Lee \& Hughes, 2004). These thinking patterns are erroneous and are known as distortions because they are baseless and without any concrete supportive evidence (Stewart, Kennard, Lee \& Hughes, 2004).Cognitive distortions are often accompanied with dysthymic mood in clinical and non-referred young sample (Stewart, Kennard, Lee \& Hughes, 2004). Thinking constantly in distorted ways can result in different psychopathological behaviours for example internalising behaviours including but not limited to depression and anxiety, as well as externalising behaviours for example antisocial behaviour and aggression (Owens, Skrzypiec \& Wadham, 2014). Although cognitive distortions that are self-serving might play a useful role by avoiding to develop negative self-concept and inhibiting aggressive behaviour (Owens, Skrzypiec \& 
Wadham, 2014). According to Barriga et al., (2000) these self-serving cognitive distortions as an agent to neutralize guilt while according to Bandura (1991), they support moral disengagement (Owens, Skrzypiec \& Wadham, 2014; Millon, 2011).

Self-debasing cognitive distortions lead to low self-efficacy and degradation of self erroneously that result in internalizing behavioural problems and emotional difficulties (Owens, Skrzypiec \& Wadham, 2014). Beck (2001) suggests different kinds of cognitive errors or distortions in thinking for example selective abstraction, overgeneralization, magnification, catastrophizing, personalization and projection that have been studied widely in the literature (Rehna, Hanif \& Aqil, 2020).

Adolescence is a period of challenges. According to Erickson, many adolescents behaviour for example rejecting the values of parents, sexual experimentations, mood swings, trying to get autonomy, depression, not performing well in studies and drug use are considered problematic by parents and teachers (Erickson \& Friedman, 1978). As majority of these adolescents are studying in different colleges, it is deemed necessary to evaluate these thinking patterns, perceptions and behaviours to evaluate whether they can be considered normal or indicate abnormality. According to the report ofUnited Nations Development Program (UNDP) Pakistan has greatest number of youth as $64 \%$ of the population of is below the age of 30 (Faisal, Hyder \& Zaidi, 2019). Prevalence of mental disorders according to WHO are around 20\% (Alvi, Ramzan \& Khan, 2010). Therefore, this study was conducted to assess the prevalence of thinking patterns among adolescents studying in different colleges of Punjab Pakistan as adolescents represent the highest number in terms of population in Pakistan and their mental health and thinking patterns are crucial to be assessed for their future.

\section{Research Objectives}

In order to find the mental health status of adolescents of Pakistan, the current researchintended toexamine the prevalence of cognitive distortions among adolescents (age range 16-22) seeking education in different public sector colleges of Rawalpindi Division of Punjab (Pakistan). 


\section{Methodology}

\section{Study Design}

The cross-sectional research study was conducted in different public sector colleges of Punjab, Pakistan during the time period of 1-9-2018 to 306-2019. The students were of intermediate and undergraduate level in the age range of 16-22.

\section{Sample Size Estimation}

Sample size was assessed using G Power calculator using 95\% Confidence Interval (Charan \& Biswas, 2013). Enrolment record was obtained from participating colleges to estimate sample size. Participant's entry into the research was also based on their willingness to be the participant in the study. Total enrolled students in Public sector colleges of Punjab were 56679 during the session 2016-2018. Keeping the confidence interval between 5 and 1, a total sample of 1258 was calculated to be sufficient to be the participants of the study. Students were of urban, suburban and rural communities. A total of 1350 college students were approached to participate in the study, and ultimately 1258 college students were finally included after screening for missing information. A total of 18 college students refused to participate whereas, 80 students had incomplete forms. After distribution of questionnaires, detailed directions were given to the participants about how to fill the protocols of research. Any confusion of the participants were addressed. Demographic information was filled by either the researcher herself or by the participant. Participants filled the questionnaires in 30-40 minutes. Ethical Considerations were strictly followed during the process of data collection.

\section{Participants}

Participants of the study were 1258 college students (males $=443$, females $=815$ ) selected from 40 colleges of Higher Education Department Punjab and Federal Government colleges under Cantt. and the Garrison System. College students were selected from these districts keeping in view the diverse geographic and socioeconomic background of students and families in these colleges. Demographic details of the participants are mentioned in table I. 
Table 1: Demographic detail of the participants of the study $(n=1258)$

\begin{tabular}{cc}
\hline Variables & $\mathbf{f \%}$ \\
\hline Age (in years) & $3.88 \%$ \\
17 & $22.30 \%$ \\
18 & $30 \%$ \\
19 & $20.71 \%$ \\
20 & $28.71 \%$ \\
21 & $2.85 \%$ \\
22 & \\
Gender wise distribution & $35.2 \%$ \\
Males & $64.8 \%$ \\
Females & \\
Mother Language & $53.3 \%$ \\
Punjabi & $34.9 \%$ \\
Urdu & $7.1 \%$ \\
Pashto & $3.3 \%$ \\
Kashmiri & $1.3 \%$ \\
Hindko & \\
Birth Order & $36.1 \%$ \\
First Child & $29.5 \%$ \\
Second Child & $17.1 \%$ \\
Third Child & $10.2 \%$ \\
Fourth Child & $5.9 \%$ \\
Fifth Child &
\end{tabular}

\section{Measures}

"How I think Questionnaire" was used to examine cognitive distortions among college students (age range 16-22) in Pakistan. It has 54 items and is designed on a six point Likert rating scale that ranges from strongly disagree to strongly agree (Frey, 2002). There are eight sub scales of this questionnaire including Self-Centered (SC), Lying (L), Minimizing/ Mislabeling (MM), Opposition Defiance (OD), Blaming Others (BO), Stealing (S), Assuming the Worst (AW), Physical Aggression (PA) (Bogestad, Kettler \& Hagan, 2010).Two summary scale scores can also be obtained i.e., overt and covert besides having a total score. If an adolescent or a youth circles two responses to an item, higher value was scored. Clinical cutoff points for the HIT-Qin the present research study was 2.98 or higher Total Scores on the Questionnaire were intended to represent thinking errors or cognitive distortions (Barriga, Hawkins \& Kamelia, 2008). 
Scoring range of the HIT-Q is 40-240. If the participant score in lower range it is indicative of normal thinking process while high scores are indicative of distorted thinking (Bacchini, De Angelis, Affuso \& Brugman, 2016). Four subscales ofthe Questionnaire that are covered through 39 items with four subscales in each assesstwo sub dimensions. One dimension represent the four categories of antisocial behaviorwith diagnosis of oppositional defiant in the Diagnostic and Statistical Manual of Mental Disorders. Oppositional defiance also measures theft, lying and physical aggression (Ara, 2015). For instance, the item "It's no use to try to stay out of fights" signifies an assuming the worst cognitive distortion item assessing "physical aggression" (Bruno, 2010). Therefore it is assumed with certainty that the HIT-Q is an effective tool to assess externalizing behavioral problems. Other dimensions represent cognitive distortions including blaming others, minimizing/mislabeling, assuming the worst and selfcentered (DuBois, James, Chibnal \& Gibbs, 2015).

According to Wallinus et al., (2011) reliability of the HIT-Q ranged between .79-.90 for subscales of cognitive distortions. Reliability of Total HIT-Q score was calculated to be .79 in their study (Wallinius, Johansson, Larden \& Dernevik, 2011). According to Barriga et al., (2000), test-re test reliability of the Questionnaire is .91 and internal consistency estimated through Cronbach alpha ranged from .93 to .96 while the validity when correlated with externalizing problematic behaviours ranged from .47-.55 (Barriga, Landau, Stinson, Liau \& Gibbs, 2000).According to Bruno (2010), overall reliability of the Questionnaire is .94 while the alpha coefficients were the subscales ranged from .81-.86 (Bruno, 2010).

\section{Results}

Results of the study determine interesting findings regarding the distribution of thinking errors in form of cognitive distortions among youth in Pakistan. The percentage of students who scored higher on the HIT-Q and placed in the "clinical range" was calculated (Hernandez, 2017). The criteria for placing in the clinical range for the current research work was all those students who scored above $84^{\text {th }}$ percentile on any subscale of the HIT-Q (Hernandez, 2017). The percentage of students scoring higher than $84^{\text {th }}$ percentile and placed in clinical range category are mentioned in form of table. Range mentioned in the manual of the HIT-Q for the clinical category was followed. If the participant scored in the range of 3.20-4.89 on selfcentered subscale, they are placed in clinical range. Responses revealed that $17.17 \%$ (216/1258) scored high on "self-centered". 13.83\% (174/1258) were falling in the clinical category in Blaming others subscale as illustrated in table 2. Scoring range for placing in the clinical category on "blaming others" subscale was 3.15-5.00. $11.48 \%(148 / 1258)$ responses were in the clinical category on the "assuming the worst" subscale and $14.1 \%$ in the 
minimizing/ mislabeling subscale. 3.00-4.89 was the clinical range for thesubscale of minimizing/mislabeling and Clinical range for assuming the worst subscale according to manual of the HIT-Q was 3.00-4.92 (Barriga, Landau, Stinson, Liau \& Gibbs, 2000).Similarly responses of adolescents were also assessed for "behavioral referent subscales" as mentioned in table 2. $31.79 \%$ of adolescents scored high on oppositional defiance subscale of behavioral referent. $67.3 \%$ in "physical aggression" and $60.09 \%$ in "stealing". 4\% (52/1258) adolescent's responses fall in clinical category in "lying subscale" of the HIT-Q as mentioned in table 2.

Table 2: Clinical scores of participants on Cognitive Distortion Subscales, Behavioral Referent Subscales and the HIT-Q Total Score

\begin{tabular}{lcc}
\hline Subscales & Clinical & $\begin{array}{c}\text { Participant's response } \\
(\mathbf{n = 1 2 5 8})\end{array}$ \\
\hline Self -centred & $3.20-4.89$ & 216 \\
Blaming others & $3.15-5.00$ & 174 \\
Assuming the worst & $3.00-4.92$ & 148 \\
Minimizing /mislabelling & $3.00-4.89$ & 178 \\
Oppositional defiance & $3.26-4.60$ & 400 \\
Physical Aggression & $3.07-4.91$ & 847 \\
Lying & $2.59-3.09$ & 52 \\
Stealing & $2.61-4.67$ & 756 \\
HIT Summary Score & $3.03-4.79$ & 414 \\
Overt subscales & $2.39-2.73$ & 360 \\
Covert subscales & $303-4.85$ & 337 \\
\hline
\end{tabular}

\section{Gender Differences in the Scores of Cognitive Distortions}

Results revealed boys scored consistently higher on cognitive distortions subscales than girls as is illustrated through table III. Gender differences estimated through pairwise comparison revealed that boys $(M=2.60, S D=$ $.58)$ had higher mean scores as compared to girls $(M=2.55, S D=.59), d=$ .05 on the self -centered cognitive distortion. Boys also scored higher on blaming others sub dimension of cognitive distortion $(M=2.58, S D=.56)$, than girls $(M=2.50, S D=.51), d=.08$. Boys had higher mean scores on the subscale of minimizing/mislabeling $(M=2.34, S D=.52)$ than girls $(M=$ $2.28, S D=.50), d=.06$. Similarly on the subscale of assuming the worst, mean scores of boys $(M=2.39, S D=.46)$ was higher in comparison with girls $(M=2.38, S D=.456), d=.51$ as is evident from table III. 
Table 3: Gender Differences in mean and standard deviation in cognitive distortionsubscales, self-centered, assuming the worst, minimizing/mislabeling, blaming others $(n=1258)$

\begin{tabular}{lccccc}
\hline & Gender & $\mathbf{N}$ & Mean & $\begin{array}{c}\text { Standard } \\
\text { Deviation }\end{array}$ & $\begin{array}{c}\text { Standard } \\
\text { Error Mean }\end{array}$ \\
\hline \multirow{2}{*}{ Self-centered } & Boys & 815 & 2.55 & .59 & .02 \\
\multirow{3}{*}{ Blaming Others } & Girls & 443 & 2.60 & .58 & .02 \\
\multirow{3}{*}{ Assuming worst } & Boys & 815 & 2.50 & .53 & .01 \\
\multirow{4}{*}{ Minimizing/mislabeling } & Girls & 443 & 2.58 & .56 & .02 \\
& Boys & 815 & 2.37 & .45 & .016 \\
& Girls & 443 & 2.39 & .46 & .02 \\
& Boys & 815 & 2.28 & .50 & .017 \\
& Girls & 443 & 2.34 & .52 & .025 \\
\hline
\end{tabular}

Table 4: $t$ test values to describe differences between cognitive distortion subscales

\begin{tabular}{|c|c|c|c|c|c|c|}
\hline & \multicolumn{6}{|c|}{ Test Value $=0$} \\
\hline & \multirow{2}{*}{$\mathbf{T}$} & \multirow[t]{2}{*}{ Df } & \multirow{2}{*}{\multicolumn{2}{|c|}{$\mathbf{p}$}} & \multicolumn{2}{|c|}{$\begin{array}{l}\text { 95\% Confidence Interval of the } \\
\text { Difference }\end{array}$} \\
\hline & & & & & Lower & Upper \\
\hline Self-centered & 154.02 & 1257 & .000 & 2.57 & 2.53 & 2.60 \\
\hline Blaming Others & 169.01 & 1257 & .000 & 2.53 & 2.50 & 2.56 \\
\hline Assuming worst & 183.70 & 1257 & .000 & 2.38 & 2.36 & 2.41 \\
\hline Minimizing/mislabeling & 160.25 & 1257 & .000 & 2.30 & 2.28 & 2.33 \\
\hline
\end{tabular}

\section{Discussion}

The findings of the present study provide empirical evidence about the prevalence of thinking problems among adolescents in Pakistan. The results of the research work add to the literature by providing information about the severity of thinking errors in form of cognitive distortions in developing country of Pakistan who has one of the greatest number of youth in the age range of 15-29. These distorted way of thinking patterns are strong vulnerability factors for the development of internalizing and externalizing behavioral tendencies. Therefore it is very important for educationist and stakeholders in Pakistan to design educational programs focusing on development of positive and rational thinking patterns that do not lead to peculiaritiesof behavior. The sample of the study (1258 adolescents) was taken from different colleges of both urban and rural colleges of Rawalpindi division and Islamabad with diverse cultural backgrounds. However it was 
beyond the scope of present study to consider personality differences and parental attachment styles that may be aim of another study in future. There can be cultural differences as well that might be analyzed in future research. The results of the current study further revealed gender differences on scores of subscales of the HIT-Q. Mean score of boys was consistently higher on all the subscales of cognitive distortion than girls. The results of the current study compliment the findings by Bruno (2010) and Rehna (2017). However another study by Robenson and Alloyet al. (2013) revealed that females display more negative consequences based inferences than males (Robinson \& Alloy, 2013). Adolescence is a difficult time period in Pakistan with different physical social psychological problems and needs. Daily hassles and negative events in the life of adolescents (who mainly belong to challenging backgrounds) might be a factor behind huge percentage of students suffering from different kinds of cognitive distortions. Poor relationships irritability and aggressive tendencies are other reasons and consequences of erroneous way of thinking as was observed and reported by adolescents during the process of data collection.

College students are assets of any country. But youth in our country is facing many problems both in terms of thinking and behavior. Therefore identifying errors in thinking of youth can help in both gaining knowledge about how the youth thinks and what kind of faultsare there in their thinking process. Secondly identifying behavioral problems in youth would be helpful in finding out how they can be improved either through psychotherapy or psychoeducation or both so that future of Pakistan isstable. This study is helpful in extending the literature as there is no other study in Pakistan that have assessed cognitive distortions in young college students (16-22) Alarmingly high rate of cognitive distortions indicated in this study among Pakistani adolescents studying in different colleges of Pakistan imply the need to assess and address these issues of thinking problems. Findings are alarming in context of high frequency of cognitive distortions among large sample (1258) who completed the HIT-Q.Considering the sensitive age range, serious steps are needed to be taken by government and educationist to work on improving the thinking patterns among youth in Pakistan.This is so becausefaulty thinking problems can lead to different behavioral problems for example depression, anxiety, antisocial behaviors and violence. Deprivation and violent behaviors among the families and societies in Pakistan might be another factor behind the high frequency of cognitive distortions among adolescents in Pakistan that needs to be explored. Research evidence has been previously established among the association between cognitive distortions and anti-social behavior among high school boys according to a study by Barriga et al., (2000) (Owens, Skrzypiec, \& Wadham, 2014). Exploring these behaviors in families will further help to identify the root cause behind erroneous way of thinking in adolescents. 


\section{Conclusion}

It can be concluded that cognitive distortions seems to be prevalent at an alarming rate in public sector colleges of Punjab Pakistan. Many of these abnormal thinking patterns are learnt from their respective environment and are considered normal by most students. These erroneous way of thinking pattern are endemic and contagious. They can play havoc as they might lead to emotional pain and different externalizing and internalizing behavioral problems There is dire need to implement proper counseling and interventional procedures to improve thinking patterns of adolescents so that they can contribute in a healthy way to society and country at large.

\section{Implications}

The use of a large college students sample of adolescents, which fills a gap in the extant literature by providing generalizable results to a broader population base than community based population, mentally ill population or referred and incarcerated youth. Second, the inclusion of males and females college students in the examination of cognitive distortions is an important addition to the extant literature. The examination of gender effects provides a more generalizable set of findings and differs from many of the previous studies that only included males; thus providing further, in some cases new, information on gender similarities and differences in view of cognitive distortions. Interventions to address these distorted patterns of thinking are necessary at higher secondary schools and colleges to avoid escalations of mental health and behavioral problems in adolescents. As the adolescence is a period of developmental vulnerability and opportunity, teaching students how to ventilate these thoughts and develop effective coping and critical thinking skills to deal with these distorted thinking patterns would increase their chances of thinking in positive directions.

\section{References}

Alvi, T., Assad, F., Ramzan, M., \& Khan, F.A. (2010). Depression, anxiety and their associated factors among medical students. J Coll Physicians Surg Pak, 20(2), 122-6.

American Psychological Association. APA (1994). Diagnostic and statistical manual of mental disorders, 4.

Ara, E. (2015). Measuring Self-Serving Cognitive Distortions: An analysis of the Psychometric Properties of the How I think Questionnaire (HIT16-Q). The International Journal of Indian Psychology, 3(1).

Bacchini, D., De Angelis, G., Affuso, G., \& Brugman, D. (2016). The structure of self-serving cognitive distortions: A validation of the "How 
I Think" Questionnaire in a sample of Italian adolescents. Measurement and Evaluation in Counseling and Development, 49(2), 163-180.

Barriga, A.Q., Hawkins, M.A., \&Camelia, C.R. (2008). Specificity of cognitive distortions to antisocial behaviours. Criminal Behaviour and Mental Health, 18(2), 104-116.

Barriga, A.Q., Landau, J.R., Stinson, B.L., Liau, A.K., \& Gibbs, J.C. (2000). Cognitive distortion and problem behaviors in adolescents. Criminal justice and behavior, 27(1), 36-56.

Beck, A.T., \& Weishaar, M. (1989). Cognitive therapy. In Comprehensive handbook of cognitive therapy (pp. 21-36). Springer, New York, NY.

Bogestad, A.J., Kettler, R.J., \& Hagan, M.P. (2010). Evaluation of a cognitive intervention program for juvenile offenders. International Journal of Offender Therapy and Comparative Criminology, 54(4), 552-565.

Bruno, T. (2010). What are they thinking? Cognitive distortions and adolescent externalizing and internalizing problems (Doctoral dissertation, University of British Columbia).

Charan, J., \& Biswas, T. (2013). How to calculate sample size for different study designs in medical research? Indian journal of psychological medicine, 35(2), 121.

Faisal, T., Hyder, M., Brugman, D., \& Zaidi, S.S.Z. (2019). Behavioural Aspects of Youth in Pakistan. Unemployment and Entrepreneurship.

Helmond, P., Overbeek, G., Brugman, D., \& Gibbs, J.C. (2015). A metaanalysis on cognitive distortions and externalizing problem behavior: Associations, moderators, and treatment effectiveness. Criminal justice and behavior, 42(3), 245-262.

Hernandez, C. (2017). Self-Serving Cognitive Distortions, Externalizing Behaviors, and School Exclusion among Adolescents with Emotional Disturbance.

Millon, T. (2011). Assertive Styles, Denigrating Types, Sadistic Disorders: The Ads Spectrum Erich Fromm. Disorders of Personality, 616.

Owens, L., Skrzypiec, G., \& Wadham, B. (2014). Thinking patterns, victimisation and bullying among adolescents in a South Australian metropolitan secondary school. International Journal of Adolescence and Youth, 19(2), 190-202.

Rehna, T., \& Hanif, R. (2017). Verbal cognitive abilities and emotional and behavioral problems of secondary school children. Foundational University Journal of Psychology, 2(2), 20-35.

Rehna T, Hanif R, Aqeel M. (2020). Moderating Role of Gender on the Relationship between Cognitive Errors and Anxiety among Adolescents. Foundation University Journal of Psychology. 4(1):86-102.

Robinson, M.S., \& Alloy, L.B. (2003). Negative cognitive styles and stressreactive rumination interact to predict depression: A prospective study. Cognitive Therapy and research, 27(3), 275-291. 
Stewart, S.M., Kennard, B.D., Lee, P.W.H., Hughes, C.W., Mayes, T.L., Emslie, et al.(2004). A cross-cultural investigation of cognitions and depressive symptoms in adolescents. Journal of Abnormal Psychology, 113, 248-257.

Tems, C.L., Stewart, S.M., Skinner Jr, J.R., Hughes, C.W., \& Emslie, G. (1993). Cognitive distortions in depressed children and adolescents: Are they state dependent or trait like? Journal of Clinical Child Psychology, 22(3), 316-326.

Wallinius, M., Johansson, P., Larden, M., \& Dernevik, M. (2011). Selfserving cognitive distortions and antisocial behavior among adults and adolescents. Criminal justice and behavior, 38(3), 286-301.

\section{Author Note}

I, Miss Sobia Ishrat declare that this article is from my PhD Research titled "Cognitive Distortions and Behavioral Problems in Adolescents" submitted to Department of Behavioral Sciences: Fatima Jinnah Women University, Rawalpindi. 